\title{
Genetic diversity of cultivated flax based on CesA genes
}

\author{
A. Dmitriev ${ }^{1}$, T. Rozhmina ${ }^{1,2}$, G. Krasnov ${ }^{1}$, A. Snezhkina ${ }^{1}$, R. Novakovskiy ${ }^{1}$, \\ P. Kezimana ${ }^{1}$, N. Bolsheva ${ }^{1}$, O. Muravenko ${ }^{1}$, A. Kudryavtseva ${ }^{1}$, N. Melnikova ${ }^{1 *}$ \\ ${ }^{1}$ Engelhardt Institute of Molecular Biology, Russian Academy of Sciences, Moscow, Russia \\ ${ }^{2}$ All-Russian Research Institute for Flax, Torzhok, Russia \\ *e-mail:mnv-4529264@yandex.ru
}

Key words: cellulose synthase, CesA, Linum usitatissimum, flax, high-throughput sequencing, polymorphism, genetic diversity

Motivation and Aim: Cultivated flax (Linum usitatissimum L.) is a source of long bast fibres for textile industry, therefore, breeding for optimization of stem content in cellulose-rich bast fibres is conducted. Cellulose synthase genes (Ces $A$ ) play an important role in cellulose biosynthesis $[1,2]$. In our study, high-throughput sequencing of CesA genes was performed in flax cultivars and lines with varied cellulose content to evaluate genetic diversity of these genes.

Methods and Algorithms: DNA from 48 flax cultivars and lines with known cellulose content from the collection of the All-Russian Research Institute for Flax was extracted. DNA libraries for high-throughput sequencing of CesA genes on Illumina platform were prepared using two-stage PCR with primers designed by us. Obtained DNA libraries were quantified on Qubit 2.0 fluorometer, evaluated on Agilent 2100 Bioanalyzer, normalized, pooled, and sequenced on Illumina platform. For data analysis, CLC Genomics Workbench was used. Reads were mapped on reference sequences of Ces $A$ genes and SNP detection was performed.

Results: High-throughput sequencing of amplicons on Illumina platform allowed us to obtain thousands of reads related to Ces $A$ genes for each flax genotype that is important for proper polymorphism identification. Single nucleotide polymorphisms (SNPs) were revealed in studied cultivars and lines with varied cellulose content, and the assessment of their genetic diversity was performed.

Conclusion: In the present work, high-throughput sequencing of cellulose synthase genes was performed and polymorphisms within 48 flax cultivars and lines were identified. Our study is important for evaluation of polymorphism of CesA genes in cultivated flax, and contributes to determination of associations between particular CesA alleles and cellulose content that can be used in marker-assisted breeding.

Acknowledgements: This study was funded by the Presidium of the Russian academy of sciences, Program No. 41 "Biodiveristy of natural systems and biological resources of Russia”.

\section{References}

1. Chantreau M. et al. (2015) Functional analyses of cellulose synthase genes in flax (Linum usitatissimum) by virus-induced gene silencing. Plant Biotechnology Journal. 13(9):1312-1324.

2. Galinousky D. et al. (2017) Expression analysis of cellulose synthase and main cytoskeletal protein genes in flax (Linum usitatissimum L.). Cell Biology International. DOI: 10.1002/cbin.10837. 